\title{
Power Analysis of a 32-bit Embedded Microcontroller
}

\author{
VIVEK TIWARI ${ }^{\mathrm{a}, *}$ and MIKE TIEN-CHIEN LEE ${ }^{\mathrm{b}}$ \\ ${ }^{a}$ Dept. of Electrical Engineering, Princeton University, Princeton, NJ 08544; \\ ${ }^{\mathrm{b}}$ Fujitsu Laboratories of America, 77 Rio Robles, San Jose, CA 95134
}

\begin{abstract}
A new approach for power analysis of microprocessors has recently been proposed [14]. The idea is to look at the power consumption in a microprocessor from the point of view of the actual software executing on the processor. The basic component of this approach is a measurement based, instruction-level power analysis technique. The technique allows for the development of an instruction-level power model for the given processor, which can be used to evaluate software in terms of the power consumption, and for exploring the optimization of software for lower power. This paper describes the application of this technique for a comprehensive instruction-level power analysis of a commercial 32-bit RISC-based embedded microcontroller. The salient results of the analysis and the basic instruction-level power model are described. Interesting observations and insights based on the results are also presented. Such an instruction-level power analysis can provide cues as to what optimizations in the micro-architecture design of the processor would lead to the most effective power savings in actual software applications. Wherever the results indicate such optimizations, they have been discussed. Furthermore, ideas for low power software design, as suggested by the results, are described in this paper as well.
\end{abstract}

Keywords: Embedded software, embedded systems, low power design, low power software, power estimation, power optimization

\section{INTRODUCTION}

A very large fraction of the applications in all segments of the electronics industry are being implemented as embedded computer systems. The basic characteristic of these systems is the presence of both a hardware and a software component. The hardware component consists of applicationspecific circuits, while the software component consists of application-specific software running on dedicated microprocessors. The role of the software component is actually projected to grow in the future. A large number of embedded computing applications are power critical, i.e., power constraints form an important part of the design specification. In light of the growing role of the software component, it is imperative to consider the power consumption of this component when analyzing the total system power consumption.

In spite of its importance, very little previous work exists for analyzing power consumption

*Corresponding author. 
from the point of view of software. Some attempts in this direction are based on architectural level analysis of microprocessors. The underlying idea is to assign power costs to architectural modules such as datapath execution units, control units, and memory elements. In $[10,15]$ the power cost of a module is given by the estimated average capacitance that would switch when the given module is activated. More sophisticated statistical power models are used in [5, 6]. Activity factors for the modules are then obtained from functional simulation over typical input streams. Power costs are assigned to individual modules, in isolation from one another. Thus, these methods ignore the correlations between the activities of different modules during execution of real programs.

Since the above techniques work at higher levels of abstraction, the power estimates they provide are not very accurate. For greater accuracy, one has to use power analysis tools that work at lower levels of the design - physical, circuit, or switch level $[8,9,4]$. However, these tools are slow and impractical for analyzing the total power consumption of a microprocessor as it executes entire programs. These tools also require the availability of lower level circuit details of microprocessors, something that most embedded system designers do not have access too. This is also the reason why the power contribution of software and the potential for power reduction through software modification has either been overlooked or is not fully understood.

A recent work [14] overcomes these deficiencies by developing a methodology that provides a means for analyzing the power consumption of a given microprocessor as it executes a given program. The idea is to use a measurement based analysis technique for developing and validating an instruction level power model for any given processor. Such a model can then be provided by the processor vendors for both off-the-shelf processors, as well as embedded cores. This can then be used to evaluate embedded software, much as gate level power models have been used to evaluate logic designs. The ability to evaluate software in terms of the power metric helps in verifying if a design meets its specified power constraints. In addition, it can also be used to search the design space in software power optimization [13].

The initial work in this direction has been in the context of the Intel 486DX2, a general-purpose CISC architecture. This paper describes the application of this power analysis methodology for the Fujitsu SPARClite MB86934, a 32-bit RISC microcontroller [1-3] targeted for embedded applications. A comprehensive power analysis of this processor has been performed and an instruction level power model has been developed. The salient results of the analysis are described here. Interesting observations and insights based on the results are also presented. The successful application of the analysis methodology for two different processors provides validation for the general applicability of this methodology. This is reinforced by a recent work based on the application of this analysis technique for a specialized embedded DSP processor [7].

\section{PROCESSOR OVERVIEW}

The SPARClite MB86934 is a SPARC-based microprocessor optimized for use in embedded applications. A full description of the SPARClite family and of MB86934 (referred to as the '934 from here on) is available from other references [1-3]. However, some of the features that are relevant for the remainder of this paper are briefly mentioned below:

- Technology: 0.5 micron, 3 level metal CMOS technology. There are three separate power pins for the on-chip phase locked loop (PLL), internal logic, and $I / O$, respectively. All power supply connections can be at $3.3 \mathrm{~V}$.

- On-chip floating point unit (FPU): A highperformance on-chip FPU executes single/double precision operations.

- On-chip FIFOs: FPU instructions can get their operands from a 32-bit FPU register file, or 6 
on-chip FIFOs, which are fed directly from main memory through DMA.

- On-chip caches: A $8 \mathrm{~K}, 32$-byte line, instruction cache, and a $2 \mathrm{~K}$, 16-byte line, data cache. Both caches are 2-way set associative and employ a write-through policy and a LRU replacement algorithm. Cache entries can be locked and the caches can also be disabled.

- Large integer register file: The integer register file consists of 136, 32-bit registers, which are organized into 8 overlapping windows.

- Software controlled power management: A software mechanism is provided to disable the clocks to various functional units in order to conserve power.

\section{EXPERIMENTAL METHOD}

The instruction level power analysis technique relies on the ability to measure the average current drawn by the processor. This is motivated by the formulas for the power and energy cost of a program. The average power, $P$, consumed by a microprocessor while running a certain program is given by: $P=I \times V_{C C}$, where $I$ is the average current, and $V_{C C}$ is the supply voltage. Since power is the rate at which energy is consumed, the energy, $E$, consumed by a program is given by: $E=P \times T$, where $T$ is the execution time of the program. This in turn is given by: $T=N \times \tau$, where $N$ is the number of clock cycles taken by the program, and $\tau$ is the clock period.

For the experimental setup used in this study, $V_{C C}$ was $3.3 \mathrm{~V}$ and $\tau$ was $50 \mathrm{~ns}$, corresponding to the $20 \mathrm{MHz}$ systems clock. Thus, if the average current for an instruction sequence is $I$ Amperes, and the number of cycles it takes to execute is $N$, the energy cost of the sequence is given by: $E=I \times$ $N \times \tau$, which equal: $\left(16.5 \times 10^{-8} \times I \times N\right)$ Joules. Throughout the rest of the paper, in order to specify the energy cost of an instruction (instruction sequence), the average current will be specified. The number of cycles will either be explicitly specified, or will be clear from the context.

\subsection{Current Measurement}

From the above discussion it is evident that to measure the energy cost of a program, the average current drawn by the CPU during the execution of the program has to be measured. The measurement method employed was based on the test and measurement capabilities of a commercial IC tester. The program under consideration was first simulated on a VERILOG model of the CPU. This produces a trace file consisting of vectors that specify the exact logic values that would appear on the pins of the CPU for each half-cycle during the execution of the program. The tester then applies the voltage levels specified by the vectors on each input pin of the CPU. This recreates the same electrical environment that the CPU would see on a real board. The current drawn by the CPU is monitored by the tester using an internal digital ammeter. Now, the current drawn by the CPU varies over the execution of a program, and so the ammeter may not yield a steady visual reading. To overcome this, the method used in the case of the 486DX2 is applied [14]. The programs being considered are put in infinite loops. Thus, the resulting current waveforms are now periodic. The ammeter averages current over a window of time (about $100 \mathrm{~ms}$ ) for the purpose of analog to digital coversion. If the period of the current waveform is much smaller than this window, a stable reading is obtained.

\subsection{Instruction Level Power Analysis}

The above method makes it feasible to measure the power cost of a given program. By designing special programs and measuring their power cost, it is possible to obtain the basic information needed for an instruction level power analysis of the processor, based on the following hypothesis. Consider a program consisting of several instances of a certain instruction. Since the CPU is executing the same instruction over and over again, it seems intuitive that the entire activity in the CPU can be attributed to that instruction. The power cost of 
the CPU for the program can be considered as the basic power cost of the given instruction. In real programs there may be other effects involving more than one instruction that can impact the power cost, e.g., the effect of circuit state, pipeline stalls, and cache misses. By designing programs where these effects occur repeatedly, can similarly provide a way for assigning power costs to these effects too.

This hypothesis has been validated for the Intel 486DX2. It has also been found to be applicable for the ' 934 , as the subsequent sections will show. The instruction level power model that has been developed for the two processors has the same basic components. The first of these is the set of base costs of instructions. The base cost of a given instruction is obtained by creating a program consisting of several instances of the instruction executing in a loop. The other component of the power model is the power cost of inter-instruction effects. The first of these is the effect of change of circuit state between consecutive instructions. During determination of the base costs, the same instruction is executed again and again. It can be expected that the change in circuit state between consecutive instructions will be less here, than for the case in which consecutive instructions differ. The quantity circuit state overhead is introduced to account for this effect. This effect is illustrated in some of the later sections and is discussed in detail in Section 10. The overall instruction-level power model and its use in estimating the power consumption of programs is described in Section 11.

\section{POWER ANALYSIS OF THE '934}

In the subsequent sections, the specifics of the instruction level power model for the ' 934 are presented. Other results that highlight the characteristics of the power consumption, as it relates to instructions and software, are also reported. For the sake of clarity, the experiments are divided into several categories, each of which is treated in a separate section. The results include the power costs of the important instructions, and examples that illustrate the power model that is used for the estimation of power consumption of instruction sequences. The power costs of external memory accesses, and the effect of the caches on the overall power cost is also explored. Results are also provided for the impact of software controlled power management on the power cost of instructions. The salient observations and interesting insights based on the results of each section are also briefly discussed following the results. One of the benefits of an instruction-level analysis is that it provides cues as to what optimizations in the micro-architecture design would lead to the most effective power savings in actual software applications. Wherever the results indicate such optimizations, they have been discussed. Furthermore, ideas for low power software design, as suggested by the results, are also described.

The following observations are valid for all experiments reported in this paper. Repeated runs of an experiment at different times resulted in only a very small variation in the observed average current values. The variation was in the rage $\pm 1 \mathrm{~mA}$. The current drawn by the power pin connected to the onchip PLL was very small and below the measuring range of the tester. The current drawn by the power pins connected to the internal logic and $I / O$ circuitry is denoted by $I 1$ and $I 2$, respectively. The symbol ' $\&$ ' used in the tables below for an instruction pair ' $i \& j$ ' denotes an instruction sequence where instructions $i$ and $j$ are executed alternately. Instructions are specified using the standared SPARC assembly language syntax [2]. In particular, $\%$ xi refers to the $i$ th register of type $x$, [\% xi] refers to the contents of the memory location that is addressed through register $\% \mathrm{xi}$, and the destination operand is always specified as the rightmost operand in an instruction.

\section{INTEGER ALU INSTRUCTIONS: CACHES ENABLED}

Table I shows the base costs for some integer instructions. The caches, prefetch, and write buffers are enabled. These modules can be enabled 
TABLE I Sample integer ALU instructions: caches enabled

\begin{tabular}{|c|c|c|c|c|}
\hline No. & Instruction & Register contents & $I 1(\mathrm{~mA})$ & $I 2(\mathrm{~mA})$ \\
\hline 1 & or $\% \mathrm{~g} 0,0, \% 10$ & & 177 & 21 \\
\hline 2 & or $\% \mathrm{~g} 0,0 \mathrm{xfff}, \% 10$ & & 174.5 & 21 \\
\hline 3 & or $\% \mathrm{~g} 0, \% \mathrm{i} 0, \% 10$ & $(\% \mathrm{i} 0=0)$ & 177.5 & 21 \\
\hline 4 & or $\% \mathrm{~g} 0, \% \mathrm{i} 0, \% 10$ & $(\% \mathrm{i} 0=0 \mathrm{xfff})$ & 173.5 & 21 \\
\hline 5 & add $\% \mathrm{i} 0, \% \circ 0, \% 10$ & $(\% \mathrm{i} 0=0, \% \mathrm{o} 0=0)$ & 178 & 21 \\
\hline 6 & add $\% \mathrm{i} 0, \% \circ 0, \% 10$ & $(\% \mathrm{i} 0=0, \% \circ 0=0 x \mathrm{xff})$ & 174 & 21 \\
\hline 7 & add $\% \mathrm{i} 0, \% \circ 0, \% 10$ & $(\% \mathrm{i} 0=0 x \mathrm{xff}, \% \mathrm{o} 0=0)$ & 174 & 21 \\
\hline 8 & add $\% \mathrm{i} 0, \% \circ 0, \% 10$ & $(\% \mathrm{i} 0=0 \mathrm{xfff}, \% \mathrm{o} 0=0 \mathrm{xfff})$ & 173 & 21 \\
\hline 9 & add $\% \circ 0, \% \mathrm{i} 1, \% 12$ & $(\% \mathrm{o} 0=0, \% \mathrm{il}=0 \times 555)$ & 174.5 & 21 \\
\hline 10 & sr1 $\% \mathrm{i} 0, \% \mathrm{o} 0, \% 10$ & $(\% \mathrm{i} 0=0 \mathrm{xfff}, \% \mathrm{o} 0=0 \times 1)$ & 179 & 21 \\
\hline 11 & sr1 \%i0, 1, \%10 & $(\% \mathrm{i} 0=0 \mathrm{xfff})$ & 176 & 21 \\
\hline 12 & sr1 \% $11, \% \circ 5, \% \mathrm{~g} 3$ & $(\% \mathrm{i} 0=0 \times 555, \% \circ 5=1)$ & 174.5 & 21 \\
\hline 13 & or $\% \mathrm{~g} 0, \% \mathrm{r} 16, \% \mathrm{i} 0$ & $(\% \mathrm{r} 16=0)$ & 178 & 21 \\
\hline 14 & orcc $\% \mathrm{i} 1, \% \mathrm{o} 0, \% 11$ & $(\% \mathrm{i} 1=0 \times 555, \% \circ 0=0 \times a a a)$ & 173 & 21 \\
\hline 15 & subx $\% \mathrm{~g} 0, \% \mathrm{r} 16, \% \mathrm{i} 0$ & $(\% \mathrm{r} 16=0)$ & 172 & 21 \\
\hline 16 & xor $\% \mathrm{~g} 0, \% \mathrm{r} 16, \% \mathrm{i} 0$ & $(\% \mathrm{r} 16=0)$ & 177.5 & 21 \\
\hline 17 & xor $\% \mathrm{~g} 0, \% \mathrm{r} 17, \% \mathrm{i} 0$ & $(\% \mathrm{r} 17=0)$ & 176 & 21 \\
\hline 18 & andcc $\% g 1,0 x a a a, \% 10$ & $(\% \mathrm{~g} 1=0 \times 555)$ & 179 & 21 \\
\hline 19 & s11\%०4, 0x7, \%०6 & $(\% \circ 4=0 \times f 0)$ & 173.5 & 21 \\
\hline 20 & umul $\% \mathrm{i} 0,0 \times 2, \% \circ 3$ & $(\% \mathrm{i} 0=0 \times a a a)$ & 174.5 & 21 \\
\hline 21 & mul \%g0, \%r29, \%r27 & $(\% \mathrm{r} 29=0)$ & 177 & 21 \\
\hline
\end{tabular}

or disabled by writing into a specific system control register. The base costs are shown in terms of the $I 1$ and $I 2$ current. All the instructions shown execute in one cycle, except entry 20 , which executes in 2 cycles.

\section{Observations and Comments}

Integer ALU instructions tend to have very similar costs, as shown in the above table. They vary in the range of $170-180 \mathrm{~mA}$, in terms of $I 1$ current. $I 2$ current is mostly stable around $21 \mathrm{~mA}$. The reason for the low $I 2$ current is that the caches are enabled, and thus, after one iteration of the loop, the instructions are always available in the instruction cache, and there is no traffic on the $I / O$ pins.

The $I 1$ current shows a limited variation depending upon the actual value of the data operands used. Variation due to the use of different registers is not significant. Entries 1 and 2 show the cost for an OR instruction for two different immediate operand values. Entries 3 and 4 show the costs for the OR instruction when only register operands are used, but the content of one of the registers is different. Entries 5 to 9 show the costs for an ADD instruction for different combinations of the operands. There seems to be a correlation between the number of 1 's in the binary representation of operands and the base cost-more the 1's, lesser the cost. The reason for the correlation has to do with the underlying circuit style used for implementing the datapath modules and busses. In any case, the overall range of variation is very limited, and thus the use of average base costs for instructions should suffice for program energy estimation purposes. This in fact is the only option in cases where the exact value of operands cannot be determined until runtime.

- An interesting observation leading from the above results is that the cost of the ALU instructions doesn't seem depend much on the ALU operation that is being performed. The cost of an OR, SHIFT, ADD, or MULTIPLY all seems to be about the same. It may well be the case that the differences in the circuit activity for these instructions are much less relative to the circuit activity common to all instructions. Thus, these differences are not reflected in the comparisons of the overall current cost. Nevertheless, the almost complete lack of variation is 
somewhat counter-intuitive. For instance, it is expected that the logic for an OR should be much less than that for an ADD, thus leading to some variation in the overall current drawn for these instructions.

The reason for the similarity of the costs most likely has to do with the way ALUs are traditionally designed. All the different ALU subfunctions are fed by a common bank of inputs, and the outputs of the appropriate sub-function are selected by a multiplexor structure. Now, in any given cycle, the results of only one subfunction are needed. Thus, the circuit activity in the other sub-functions is a waste of power. The design can be modified for low power by extending the principles of automatic power management. If the inputs of the sub-functions that are not needed are prevented from switching, the power consumed in these sub-functions can be saved. This observation motivates the concept of guarded evaluation, which has been explored in detail in another reference [12].

\section{INTEGER ALU INSTRUCTIONS: CACHES DISABLED}

Table II shows some of the same instructions as the previous table. However, in this case, the onchip caches have been disabled. Prefetch and write buffers are also disabled. The number of memory wait states is zero. Column 4 shows $I 1$ current in this case. The $I 2$ current was $134 \mathrm{~mA}$ for all entries. Column 5 shows the $I 1$ current for the case when the caches are enabled. The $I 2$ current in this case was $21 \mathrm{~mA}$.

\section{Observation and Comments}

Since the instruction cache is disabled, every instruction access goes to the external $I / O$ pins. The $I 2$ current is therefore higher than when caches are enabled. The $I 1$ current (internal logic current) is also about $10 \mathrm{~mA}$ higher. Entries 3 and 6 show what happens when different instructions are executed together. This will be discussed in greater detail in Section 10.

In terms of overall current, disabling the instruction cache leads to a total CPU current increase of about $123(=10+(134-21)) \mathrm{mA}$, i.e., about $62 \%$. However, when the cache is disabled in the ' 934 , every instruction fetch takes two cycles, even for a zero wait state system. Thus, in terms of energy, disabling the instruction cache leads to at least about a $124 \%(=2 \times 62 \%)$ increase in the energy consumption. This points to two things:

- Accessing the cache is much more energy efficient than accessing external memory. Thus, attempts to increase the cache hit rate through software modifications will be very beneficial. It is further indicated that attempts to increase the hit rate through architectural transformations may also help reduce the overall energy consumption.

- In certain embedded applications, the designer may choose to disable the caches. This is usually

TABLE II Integer ALU instructions: caches disabled vs. enabled

\begin{tabular}{lllcc}
\hline No. & Instruction & Register contents & $\begin{array}{c}\text { disabled } \\
I 1(\mathrm{~mA})\end{array}$ & $\begin{array}{c}\text { enabled } \\
I 1(\mathrm{~mA})\end{array}$ \\
\hline 1 & or $\% \mathrm{~g} 0,0, \% 10$ & & 187.5 & 177 \\
2 & or $\% \mathrm{~g} 0,0 \mathrm{xfff} \% 10$ & & 184 & 174.5 \\
3 & $1 \& 2$ & $(\% \mathrm{i} 0=0)$ & 196 & 192 \\
4 & or $\% \mathrm{~g} 0, \% \mathrm{i} 0, \% 10$ & $(\% \mathrm{i} 0=0 \mathrm{xfff})$ & 188 & 177.5 \\
5 & or $\% \mathrm{~g} 0, \% \mathrm{i} 0, \% 10$ & & 184 & 173.5 \\
6 & $4 \& 5$ & $(\% \mathrm{i} 0=0 \mathrm{xfff}, \% \mathrm{o} 0=0 \mathrm{x} 1)$ & 182 & 187.5 \\
7 & sr $\% \mathrm{i} 0, \% \mathrm{o} 0 \% 10$ & 184 & 179 \\
8 & sr $\% \mathrm{i} 0,1, \% 10$ & $(\% \mathrm{i} 0=0 \mathrm{xfff})$ & 176 \\
\hline
\end{tabular}


done to improve the performance predictability for real-time systems. However, this will lead to a penalty in terms of the system energy consumption, and thus, the battery life. This fact has to be understood and weighed in, when deciding on whether the caches should be disabled.

\section{LOAD AND STORE INSTRUCTIONS: CACHES ENABLED AND LOCKED}

Table III shows the cost of some instructions that reference memory. Since the ' 934 is a RISC, loadstore machine, the only instructions that explicitly reference memory are the loads and the stores. The above results are for the specific case when the caches are active and the entries in the data cache are locked. This implies that every data access is a cache hit. In addition, since the cache entries are locked, the store (write) instructions also don't go out to external memory. Note that the ' 934 has a write-through cache, and thus in the normal case, each data write also goes out to the external bus.
Since there is no traffic on the $I / O$ pins, the $I 2$ current is low. Each instruction also executes in one cycle in this case.

\section{Observations and Comments}

Entries 1 and 2 are direct loads and entries 10 and 11 are direct stores. The rest of the instructions utilize the indirect addressing modes. The results indicate that there is not much difference between these two addressing modes, in terms of base current. Entries 3 to 6 show the variation in the cost of a load for a fixed address but differeing data operands. Entries 3, 7, 8, and 9 show the variation for a fixed data operand but differing addresses. Entries 12, 13, and 12, 14, show the corresponding variation in the case of stores. The general trend points to a correlation between base cost and the number of 1 's in the binary representation of the data operand and the memory address. This is similar to what was seen in Section 5 - more the 1's, lower the cost. The variation in the costs, though, is again limited. Entries 16 to 23 show what happens when different

TABLE III Load and store instructions: caches enabled and locked

\begin{tabular}{|c|c|c|c|c|}
\hline No. & Instruction & Register contents & $I 1(\mathrm{~mA})$ & $I 2(\mathrm{~mA})$ \\
\hline 1 & ld $[0 \times 0], \% \mathrm{i} 0$ & $(\% \mathrm{i} 0=0)$ & 191.5 & 21 \\
\hline 2 & ld [0xffc], \%i0 & $(\% \mathrm{i} 0=0)$ & 187 & 21 \\
\hline 3 & ld [\%10], \%i0 & $(\% \mathrm{i} 0=0, \% 10=0)$ & 192 & 21 \\
\hline 4 & ld $[\% 10], \% \mathrm{i} 0$ & $(\% \mathrm{i} 0=0 x \mathrm{xff}, \% 10=0)$ & 189.5 & 21 \\
\hline 5 & ld [\%10], \%i0 & $(\% \mathrm{i} 0=0 x \mathrm{ffffff}, \% 10=0)$ & 187.5 & 21 \\
\hline 6 & ld [\%10], \%i0 & $(\% \mathrm{i} 0=0 x$ xfffffff, $\% 10=0)$ & 185 & 21 \\
\hline 7 & ld $[\% 10], \% \mathrm{i} 0$ & $(\% \mathrm{i} 0=0, \% 10=0 x f f c)$ & 191 & 21 \\
\hline 8 & ld $[\% 10], \% \mathrm{i} 0$ & $(\% \mathrm{i} 0=0, \% 10=0 x \mathrm{fffffc})$ & 188 & 21 \\
\hline 9 & ld [\%10], \%i0 & $(\% \mathrm{i} 0=0, \% 10=0 x$ ffffffff $)$ & 185 & 21 \\
\hline 10 & st $\% \mathrm{i} 0,[0 \times 0]$ & $(\% \mathrm{i} 0=0)$ & 173 & 21 \\
\hline 11 & st $\% \mathrm{i} 0,[0 \mathrm{xffc}]$ & $(\% \mathrm{i} 0=0)$ & 169 & 21 \\
\hline 12 & st $\% \mathrm{i} 0,[\% 10]$ & $(\% \mathrm{i} 0=0, \% 10=0)$ & 175 & 21 \\
\hline 13 & st $\% \mathrm{i} 0,[\% 10]$ & $(\% \mathrm{i} 0=0 x \mathrm{xff}, \% 10=0)$ & 173.5 & 21 \\
\hline 14 & st $\% \mathrm{i} 0,[\% 10]$ & $(\% \mathrm{i} 0=0, \% 10=0 \mathrm{xffc})$ & 172 & 21 \\
\hline 15 & ldub $[\% 10], \%$ i5 & $(\% \mathrm{i} 5=0 \times a a a, \% 10=0)$ & 192.5 & 21 \\
\hline 16 & $3 \& 4$ & & 206 & 21 \\
\hline 17 & $3 \& 5$ & & 213 & 21 \\
\hline 18 & $3 \& 6$ & & 216 & 21 \\
\hline 19 & $3 \& 7$ & & 202.5 & 21 \\
\hline 20 & $3 \& 8$ & & 207 & 21 \\
\hline 21 & $3 \& 9$ & & 211 & 21 \\
\hline 22 & $12 \& 13$ & & 185 & 21 \\
\hline 23 & $12 \& 14$ & & 183 & 21 \\
\hline
\end{tabular}


instructions execute together. The data and address registers used for each instruction in the pair were different, but the register contents were the same as shown in the individual instruction entries. Entry 16 is for the case when the instructions in entry 3 and 4 execute alternately. The current is higher than the average of the two base costs. This is due to the effect of circuit state overhead. 12 data operand bits flip between entries 3 and 4. The entries 17 and 18 show the results for greater data flips. Entries 19 to 20 show the results when the address bits flip between adjacent instructions. The results indicate a positive correlation between the number of bit flips, and the increased effect of circuit state.

The results also lead to the following interesting observations:

- A comparison between Tables II and IV shows that cache accesses aren't much more costly than register accesses. Cache reads are bout 10 $\mathrm{mA}$ more costly, and cache writes are about the same cost as register accesses. Since both cache and register accesses take one cycle, the energy comparison shows the same relation. This observation is in stark contrast to what was observed in the case of the Intel 486DX2, where cache accesses were much more costly than register accesses. The reason for the similarity in the cost of cache accesses and register accesses in '934 is most likely due to the large size of its register file. The ' 934 is a RISC, load-store architecture, and it is characteristic for this architectural style to use a large number of registers. The register file has 136 registers. In addition, it is multiported, and is windowed. In constrast the 486DX2 has a simple register file with only 8 registers.

This observation illustrates an interesting CISC vs. RISC trade-off with regards to power. On one hand, the availability of a larger number of registers can help reduce the use of memory operands, leading to power reductions. But on the other hand, the larger register file causes each register access itself to be costlier.

- The data also points to the fact that microarchitectural or circuit transformations to optimize the register file for low power will be very beneficial in terms of overall power reductions. The load-store design of ther '934 involves very heavy usage of the registers, and a lower power cost of accessing registers will translate into power reductions for all programs.

- It should be noted that the use of memory operands does have a high cost even in the ' 934 , due to the possibility of cache misses. Also, if the cache is unlocked, stores will incur additional cost in terms of $I 2$ current (as shown in the next section), and memory system current. Thus, the use of memory operands should certainly be avoided. This also points out that the cache locking feature should be exploited as

TABLE IV Store instructions: caches enabled and unlocked

\begin{tabular}{|c|c|c|c|c|}
\hline No. & Instruction & Register contents & $I 1(\mathrm{~mA})$ & $I 2(\mathrm{~mA})$ \\
\hline 1 & st $\% \mathrm{i0},[\% 10]$ & $(\% \mathrm{i} 0=0, \% 10=0)$ & 198 & 148 \\
\hline 2 & st $\% \mathrm{i} 0,[\% 10]$ & $(\% \mathrm{i} 0=0, \% 10=0 x f f c)$ & 191 & 115 \\
\hline 3 & st $\% \mathrm{i} 0,[\% 10]$ & $(\% \mathrm{i} 0=0, \% 10=0$ xfffffc $)$ & 185 & 71 \\
\hline 4 & st $\% \mathrm{i} 0,[\% 10]$ & $(\% \mathrm{i} 0=0, \% 10=0 x$ fffffffc $)$ & 181.5 & 46 \\
\hline 5 & $1 \& 2$ & & 198 & 137 \\
\hline 6 & $1 \& 3$ & & 199 & 116 \\
\hline 7 & $1 \& 4$ & & 200 & 106 \\
\hline 8 & st $\% \mathrm{i} 0,[\% 10]$ & $(\% \mathrm{i} 0=0 x$ fff, $\% 10=0)$ & 193 & 148 \\
\hline 9 & st $\% \mathrm{i} 0,[\% 10]$ & $(\% \mathrm{i} 0=0 x$ ffffff, $\% 10=0)$ & 191 & 150 \\
\hline 10 & st $\% \mathrm{i} 0,[\% 10]$ & $(\% \mathrm{i} 0=0$ xffffffff, $\% 10=0)$ & 189 & 150 \\
\hline 11 & $1 \& 8$ & & 203 & 173 \\
\hline 12 & $1 \& 9$ & & 207 & 193 \\
\hline 13 & $1 \& 10$ & & 211 & 206.5 \\
\hline
\end{tabular}


far as possible, for applications where energy consumption is a design constraint.

\section{STORE INSTRUCTIONS: CACHES ENABLED AND UNLOCKED}

Table IV shows the costs of some store instructions when the caches are enabled but are unlocked. Since the data cache is write-through, all the stores also reference the external main memory. The number of memory wait states is zero. However, the design of the '934 imposes an extra cycle for every bus transaction. During this cycle the bus is idle.

\section{Observations and Comments}

Most typical applications do not lock the data cache. Thus, the stores in these applications will go out to the external bus, leading to higher $I 2$ current, as shown in table. This table, therefore, reflects the more typical cost of memory writes. Note that there will also be an additional system energy penalty due to the current being drawn by the external memory.

Entries 1, 8, 9 and 10 show the variation in the cost of the stores for a fixed address but varying data. There is a minor decrease in both $I 1$ and $I 2$ current for increasing number of 1's. Entries 11 to 13 consider instruction sequences where different stores alternate. For example, entry 11 shows the cost for a sequence consisting of the instructions in entry 1 and 2 appearing in succession. The $I 1$ and I 2 currents are greater than the average of the current costs for the individual instructions. This is another illustration of the effect of circuit state overhead. The $I 1$ overhead represents the effect of the circuit state in the internal logic circuits, while the $I 2$ overhead represents the effect of switching on the data pins. Entry 11 involves 12 bit flips at the data lines, while entries 12 and 13 involve 24 and 32 bit flips respectively. As expected, greater number of bit flips, result in greater current. The increase in current is greater in the case of $I 2$ current. This too is expected, since the $I / O$ pads typically involve larger capacitive loads.

Entries 1 to 4 show the variation in the cost of stores for a fixed data value but varying addresses. The $I 1$ current decreases with an increase in the number of 1's in the binary representation of the address. The $I 2$ current also decreases, but the decrease is very drastic. For example, consider entries 1 and 4 . Entry 1 has no 1's in the address, and entry 4 has 301 's. The difference in the $I 2$ current is $102 \mathrm{~mA}$. This translates into about 3.3 $\mathrm{mA}$ per each occurrence of " 0 " in the binary representation of the address. Comparisons between the other entries also yield the same result. This observation seems strange, since if the same address is being put on the bus for every instruction, the address pins should not switch. However, the address pins do switch due to the following reason. Every memory transaction involves an extra cycle during which the bus is idle. During these bus idle cycles, the ' 934 pulls up the address pins, i.e., the pins go to the logical value 1 . Thus, even if back to back store instructions use the same address, there is an intervening cycle when the address pins are all 1's. This means that the pins corresponding to the address bits that are 0 will switch each time. More 1 's in the address value means less switching, and thus lower $I 2$ current.

Entries 5 to 7 show another illustration of this effect. The $I 2$ value for a pair of stores is about the same as the average of the $I 2$ values of the individual stores. There is no circuit state overhead. The reason being that the intervening bus idle cycle, in which the address pins are pulled up, isolated the stores from each other.

The above results lead to the following observations:

- The above results show that occurrence of 0's in the address values means greater current cost, on the order $3.3 \mathrm{~mA}$ of $I 2$ current for each occurrence of a 0 . This suggests that if data and instructions are stored at the higher end of 
memory, the program energy cost may be reduced. The reason for this is that, on the average, addresses then will have lesser 0's in them. The power reduction can potentially be very significant.

- It should be noted that the higher current cost of 0 's in the address is a manifestation of the effect of circuit state (in other words, switching) on the address pins. Now, most real systems utilize wait states, since memory access times are usually slower than the CPU clock period. If the number of wait states increases, there will be a greater number of bus idle cycles. We know that during the bus idle cycles, the address pins are at a constant 1 . Thus, more wait states means that on the average, the address pins will switch less often, leading to a lesser impact on the overall system energy cost. This is in line with the observation in the case of the 486DX2, where it was noted that for real systems, switching on the external pins had limited impact on the overall energy cost of programs.

- Switching on the data pins also leads to an increase in the current cost, though this increase is limited. While attempts to reduce this cost through software modifications may be beneficial, the benefits are likely to be very modest. This is because of the difficulty in applying these methods in general, and also because the energy impact of the switching itself is limited. This will be more so in case of real systems that have slow buses and utilize wait-states.

- A sequence of back to back stores causes the write buffer to fill up, and leads to extra cycle penalties, referred to as write buffer stalls. The cycles penalties translate into energy penalties. If the memory system is slow, and requires wait states, the number of write buffer stalls will increase. Software modifications to decrease these stalls will result in lower power. This can be done by scheduling instructions that don't require memory transactions to occur between consecutive store instructions. Specific experiments can also assign energy costs to the write buffer stall cycles, as has been done in the case of the 486DX2 [14].

\section{FLOATING POINT INSTRUCTIONS}

Table V shows the costs for some typical floating point instructions. The caches were enabled and unlocked in all experiments. The FPU is pipelined and Column 4 shows the throughput for each instruction. Column 5 shows the $I 1$ current. The I 2 current was $21 \mathrm{~mA}$ for all cases. The energy cost of an instruction is proportional to the product of the total current and the number shown in Column 4.

\section{Observation and Comments}

The results indicate that most instructions that involve the FPU have similar cost. For example, consider entries 1 to 14 , and 21 to 23, all of which take one cycle, and don't cause any FPU pipeline interlocks. The dependence of current on the value of operands is almost negligible, and this may have to do with the circuit design of the FPU. In addition, dependence of current on the type of FPU operation is also not exhibited. This may be due to the same reasons as discussed in Section 5. Instructions for loading values into floating point registers (entries 15 to 20 ) result in costs that are similar to those seen in the case of integer registers. The trend with respect to the current cost and the number of 1's in the data operands is also similar.

Entries 24 to 26 show floating point divide instructions, and entries 32 to 34 show square root instructions. The current variation for different operand values is negligible. These instructions take 13 cycles in a particular FPU pipeline stage. This leads to 12 pipeline interlocks. This means that an FPU instruction that immediately follows one of these instructions will have to wait for 12 cycles. However, the integer pipeline may not be held up in most cases, and can continue to execute. Entry 27 shows what happens when a NOP 
TABLE V Floating point instructions: caches enabled

\begin{tabular}{|c|c|c|c|c|}
\hline No. & Instruction & Register contents & $n$ & $I 1(\mathrm{~mA})$ \\
\hline 1 & fitos $\% \mathrm{f} 4, \%$ f 0 & $(\% \mathrm{f} 4=0)$ & 1 & 177.5 \\
\hline 2 & fitos $\% \mathrm{f} 4, \%$ f 0 & (\%f $4=0 x$ xff $)$ & 1 & 177.5 \\
\hline 3 & fmovs $\%$ f $4, \%$ f 0 & $(\% \mathrm{f} 4=0)$ & 1 & 175 \\
\hline 4 & fmovs $\%$ f $4, \%$ f 0 & $(\% \mathrm{f} 4=0 \mathrm{xff})$ & 1 & 175 \\
\hline 5 & fmovs $\%$ f $4, \%$ f 0 & (\%f $4=0 x f f f f)$ & 1 & 174 \\
\hline 6 & fmovs $\%$ f $4, \%$ f 0 & $(\%$ f $4=0 x f f f f)$ & 1 & 175 \\
\hline 7 & fitod $\% \mathrm{f} 4, \% \mathrm{f} 0$ & $(\% \mathrm{f} 4=0)$ & 1 & 178 \\
\hline 8 & fadds $\%$ f $8, \%$ f $4, \%$ f 0 & $(\% \mathrm{f} 8=0, \% \mathrm{f} 4=0)$ & 1 & 175.5 \\
\hline 9 & fadds $\%$ f $8, \%$ f $4, \%$ f 0 & $(\% \mathrm{f} 8=0 \mathrm{xffff}, \% \mathrm{f} 4=0 \mathrm{xffff})$ & 1 & 176 \\
\hline 10 & fadds $\%$ f $8, \%$ f $4, \%$ f 0 & $(\%$ f $8=0 \times 555555, \%$ f $4=0 \times a a a a a a)$ & 1 & 177 \\
\hline 11 & fadds $\%$ f $8, \%$ f $4, \%$ f 0 & $(\%$ f $8=0 x f f f f f f, \% f 4=0 x f f f f f)$ & 1 & 178 \\
\hline 12 & faddd $\%$ f $8, \%$ f $4, \%$ f 0 & $(\% \mathrm{f} 8=0, \% \mathrm{f} 4=0)$ & 1 & 177 \\
\hline 13 & faddd $\%$ f $8, \%$ f $4, \%$ f 0 & $(\% \mathrm{f} 8=0 \mathrm{xffff}, \% \mathrm{f} 4=0 \mathrm{xffff})$ & 1 & 177.5 \\
\hline 14 & faddd $\%$ f $8, \%$ f $4, \%$ f 0 & (\%f $8=0 \times 555555, \%$ f $4=0 \times$ xaaaaaa $)$ & 1 & 177.5 \\
\hline 15 & $1 \mathrm{~d}[0 \mathrm{x} 0], \% \mathrm{f} 8$ & $(\% \mathrm{f} 8=0)$ & 1 & 205 \\
\hline 16 & 1d [0x0], \%f 8 & $(\% \mathrm{f} 8=0 \times 4 \mathrm{~b} 7 \mathrm{ff})$ & 1 & 198 \\
\hline 17 & $1 \mathrm{~d}[0 \mathrm{x} 0], \% \mathrm{f} 8$ & $(\% \mathrm{f} 8=0 \times 4 \mathrm{~b} 7 \mathrm{fffff})$ & 1 & 193 \\
\hline 18 & $1 \mathrm{dd}[0 \mathrm{x} 0], \% \mathrm{f} 8$ & $(\% \mathrm{f} 8=0,0)$ & 1 & 214 \\
\hline 19 & $1 \mathrm{dd}[0 \mathrm{x} 0], \% \mathrm{f} 8$ & $(\%$ f $8=0 x 416 \mathrm{fffff}, \mathrm{e} 0000000)$ & 1 & 200 \\
\hline 20 & $1 \mathrm{dd}[0 \mathrm{x} 0], \%$ f 8 & $(\% \mathrm{f} 8=0 \times 4 \mathrm{~b} 7 \mathrm{fffff}, 4 \mathrm{~b} 7 \mathrm{fffff})$ & 1 & 192 \\
\hline 21 & fmuls $\%$ f $8, \%$ f $4, \%$ f 0 & $(\% \mathrm{f} 8=0, \% \mathrm{f} 4=0)$ & 1 & 174 \\
\hline 22 & fmuls $\%$ f $8, \%$ f $4, \%$ f 0 & $(\%$ f $8=0 x$ xff, $\%$ f $4=0 x f f f)$ & 1 & 175 \\
\hline 23 & fmuls $\%$ f $8, \%$ f $4, \%$ f 0 & $(\% \mathrm{f} 8=0 \times 555555, \% \mathrm{f} 4=0 \times \mathrm{xaaaaaa})$ & 1 & 175 \\
\hline 24 & fdivs $\%$ f $8, \%$ f $4, \%$ f 0 & $(\% \mathrm{f} 8=0 \times$ xaaaaa, $\% \mathrm{f} 4=0 \times 555555)$ & 13 & 167.5 \\
\hline 25 & fdivs $\%$ f $8, \%$ f $4, \%$ f 0 & $(\%$ f $8=0 x f f f, \%$ f $4=0 x$ ffff $)$ & 13 & 168 \\
\hline 26 & fdivs $\%$ f $8, \%$ f $4, \%$ f 0 & $(\% \mathrm{f} 8=0, \% \mathrm{f} 4=1)$ & 13 & 167.5 \\
\hline 27 & $26 \& 1$ nop & & 13 & 181.5 \\
\hline 28 & $26 \& 4$ nop's & & 13 & 181.5 \\
\hline 29 & $26 \& 12$ nop’s & & 13 & 182 \\
\hline 30 & $26 \& 1$ add & & 13 & 179 \\
\hline 31 & $26 \& 12$ add's & & 13 & 177.5 \\
\hline 32 & fsqrts $\% \mathrm{f} 4, \% \mathrm{f} 0$ & $(\% \mathrm{f} 4=0 \mathrm{xfe} 01)$ & 13 & 173 \\
\hline 33 & fsqrts $\% \mathrm{f} 4, \% \mathrm{f} 0$ & (\%f 4 = 0xaaaaaa) & 13 & 173.5 \\
\hline 34 & fsqrts $\%$ f $4, \%$ f 0 & $(\% \mathrm{f} 4=0)$ & 13 & 174 \\
\hline 35 & 34 \& 1 nop & & 13 & 184 \\
\hline 36 & 34 \& 4 nop’s & & 13 & 184.5 \\
\hline 37 & $34 \& 12$ nop's & & 13 & 185 \\
\hline 38 & $34 \& 1$ add & & 13 & 181 \\
\hline 39 & $34 \& 12$ add's & & 13 & 180.5 \\
\hline
\end{tabular}

instruction (internally treated as an integer instruction) appears after a divide instruction. the execution of this instruction is hidden within the 12 interlock cycles of the divide. Entries 28 to 31 show other examples when integer instructions follow a divide instruction, and entries 35 to 39 show the same for the square root instruction. These entries show that the current cost in this case isn't much more than when no integer instructions are executed in the FPU interlock cycles. This leads to the following insights:

- When integer instructions are executed during the FPU interlock cycles, the current doesn't increase much beyond what it is when the interlock cycles are completely idle. This suggests that during the FPU interlock cycles, switching activity doesn't completely stop in the other parts of the CPU. If this activity is useless, then eliminating it can result in power reduction during the interlock cycles. This represents another opportunity where automatic power management of guarded evaluation may be useful.

- The results also show that for the current implementation of the ' 934 , it is beneficial to execute integer instructions during the FPU interlock cycles. The current cost is not much 
higher than when the integer instructions are executed independently. Therefore, the decrease in the number of execution cycles translated into actual energy reduction. Execution cycles are reduced, since the cycles required to execute the integer instructions are overlapped with, and thus hidden, in the FPU interlock cycles. Software optimizations to achieve this can therfore be considered as both performance as well as energy optimizations.

\section{INTER-INSTRUCTION EFFECTS}

The previous sections mainly focussed on the base costs of instructions. The base cost of a given instruction is obtained in isolation from other instructions by repeatedly executing the same instruction. However, real programs consist of a sequence of different instructions. Several interinstruction effects can occur in these mixed sequences. Base costs themselves are not adequate to model the energy cost of these effects. These inter-instruction effects are discussed below.

\subsection{Effect of Circuit State Overhead and Instruction Reordering}

The effect of circuit state is an inter-instruction effect that has been alluded to in the previous sections. The purpose of Table VI is to illustrate and quantify this effect. Base costs of several instructions as well as the costs for pairs of instructions are shown in the table. Only the $I 1$ current is shown. The $I 2$ current was $21 \mathrm{~mA}$ in all cases. For entries with pairs of instructions, Column 4 shows the current for the combined sequence, and Column 5 shows the circuit state overhead. This value is the difference between the actual current cost of an instruction, and the average of the base costs of the individual instructions.

\section{Observations and Comments}

The existence of circuit state overhead can be attributed to the fact that each instruction executes in the context of the circuit state set by the previous instruction. The greater the change in the circuit state between instructions, greater should be this overhead. While the change in the circuit state can result from any part of the processor, the common notion is that it is basically due to the change in the opcodes of adjacent instructions. Entries 6 to 9 in Table VI show this quantitatively with an example. With increasing number of bit flips in the opcodes of adjacent instructions, the overhead increases. However, Table VI also shows that switching of the opcodes is not the only source of circuit state overhead. For example, entries 18, 19 , and 23, 24 involve almost the same number of opcode flips. However, entries 23, 24 have a much higher overhead cost.

Table VI, and some of the examples presented in Section 7, as well as in the next section, quantify several instances of circuit state overhead. These and several other examples indicate that the overhead varies between 0 and about $34 \mathrm{~mA}$. The overhead between integer instructions is typically below $20 \mathrm{~mA}$. The overhead between floating point and integer instructions is higher, typically in the range 25-34 $\mathrm{mA}$. The most important aspect of this observation is that the range of variation in this overhead is small, compared to the overall CPU current cost.

A recent idea in the area of software design for low power is to reorder instructions to reduce the power cost of a program [11]. This can be seen as an attempt to reduce the average current cost of a program by minimizing the circuit state overhead. Our experiments based on actual energy measurements on the '934, however, reveal that this technique does not translate into significant overall energy reduction. The reason is that the circuit state overhead is bounded in a small range and does not show very significant variation. Thus, different instruction schedules will not vary significantly in their current costs.

Table VII illustrates this with an example. Entries 1 to 7 show a set of instructions. Entries $a$ and $e$ show the current cost of different sequences consisting of these instructions. The order of 
TABLE VI Effect of circuit state overhead

\begin{tabular}{|c|c|c|c|c|}
\hline No. & Instruction & Register contents & $I 1(\mathrm{~mA})$ & $O v h .(\mathrm{mA})$ \\
\hline 1 & or $\% \mathrm{~g} 0,0, \% 10$ & & 177 & \\
\hline 2 & or $\% \mathrm{~g} 0,0 \times 001, \% 10$ & & 174 & \\
\hline 3 & or $\% \mathrm{~g} 0,0 \times 00 f, \% 10$ & & 174 & \\
\hline 4 & or $\% \mathrm{~g} 0,0 \mathrm{xff}, \% 10$ & & 174.5 & \\
\hline 5 & or $\% \mathrm{~g} 0,0 \mathrm{xfff}, \% 10$ & & 174.5 & \\
\hline 6 & $1 \& 2$ & 1 opcode flip & 178 & 2.5 \\
\hline 7 & $1 \& 3$ & 4 opcode flips & 184.5 & 9 \\
\hline 8 & $1 \& 4$ & 8 opcode flips & 191 & 15 \\
\hline 9 & $1 \& 5$ & 12 opcode flips & 192 & 16 \\
\hline 10 & or $\% \mathrm{~g} 0, \% \mathrm{i} 0, \% 10$ & $(\% \mathrm{i} 0=0)$ & 177.5 & \\
\hline 11 & or $\% \mathrm{~g} 0, \% \mathrm{i} 0, \% 10$ & $(\%$ io $=0 x f f f)$ & 173.5 & \\
\hline 12 & $10 \& 11$ & 12 data flips & 187.5 & 12 \\
\hline 13 & or $\% \mathrm{~g} 0, \% \mathrm{r} 16, \% \mathrm{i} 0$ & $(\% \mathrm{r} 16=0)$ & 177.5 & \\
\hline 14 & or $\% \mathrm{~g} 0, \% \mathrm{r} 17, \% \mathrm{i} 0$ & $(\% \mathrm{r} 17=0)$ & 176 & \\
\hline 15 & or $\% \mathrm{~g} 0, \% \mathrm{r} 15, \% \mathrm{i} 0$ & $(\% \mathrm{r} 15=0)$ & 175.5 & \\
\hline 16 & or $\% \mathrm{~g} 0, \% \mathrm{r} 23, \% \mathrm{i} 0$ & $(\% \mathrm{r} 23=0)$ & 176 & \\
\hline 17 & $13 \& 14$ & 1 opcode flip & 177.5 & 1 \\
\hline 18 & $13 \& 16$ & 3 opcode flips & 180 & 3 \\
\hline 19 & $13 \& 15$ & 5 opcode flips & 180.5 & 4 \\
\hline 20 & subx $\% \mathrm{~g} 0, \% \mathrm{r} 16, \% \mathrm{i} 0$ & $(\% \mathrm{r} 16=0)$ & 172 & \\
\hline 21 & xor $\% \mathrm{~g} 0, \% \mathrm{r} 16, \% \mathrm{i} 0$ & $(\% \mathrm{r} 16=0)$ & 177.5 & \\
\hline 22 & xor $\% \mathrm{~g} 0, \% \mathrm{r} 17, \% \mathrm{i} 0$ & $(\%$ r16 $=0)$ & 176 & \\
\hline 23 & $20 \& 21$ & 4 opcode flips & 191.5 & 17 \\
\hline 24 & $20 \& 22$ & 4 opcode flips & 192 & 18 \\
\hline 25 & or $\% \mathrm{il}, \% \mathrm{o} 0, \% \mathrm{ll}$ & $(\% \mathrm{il}=0 \times 555, \% \mathrm{o} 0=0)$ & 174.5 & \\
\hline 26 & add $\% \mathrm{o} 0, \% \mathrm{i} 1, \% 12$ & $(\% \mathrm{il}=0 \times 555, \% \circ 0=0)$ & 174.5 & \\
\hline 27 & or $\% \circ 5,0 \times 555, \% 14$ & $(\% \circ 5=1)$ & 170 & \\
\hline 28 & sr1 \%i1, \%o5, \%g3 & $(\% \mathrm{il}=0 \times 555, \% \circ 5=1)$ & 174.5 & \\
\hline 29 & $25 \& 26$ & & 185 & 10.5 \\
\hline 30 & $26 \& 27$ & & 182 & 9.5 \\
\hline 31 & $27 \& 28$ & & 191 & 19 \\
\hline 32 & $28 \& 25$ & & 184.5 & 10.5 \\
\hline 33 & fmuls $\%$ f8, $\%$ f4, $\%$ f0 & $(\% \mathrm{f} 8=0, \% \mathrm{f} 4=0)$ & 174 & \\
\hline 34 & nop & & 176 & \\
\hline 35 & $33 \& 34$ & & 202 & 27 \\
\hline 36 & andcc $\% g 1,0 x a a a, \% 10$ & $(\% \mathrm{~g} 1=0 \times 555)$ & 179 & \\
\hline 37 & $33 \& 36$ & & 210 & 33.5 \\
\hline 38 & ld $[0 \times 555], \% \circ 5$ & & 192.5 & \\
\hline 39 & sll \%०4, 0x7, \%०6 & $(\% \circ 4=0 \times 707)$ & 173.5 & \\
\hline 40 & $38 \& 39$ & & 202.5 & 19.5 \\
\hline 41 & or $\% \mathrm{~g} 0,0 \mathrm{xff}, \% 10$ & & 176 & \\
\hline 42 & $33 \& 41$ & & 207 & 32 \\
\hline 43 & fadd $\%$ f $10, \%$ f $12, \%$ f 14 & $\begin{array}{l}(\% \mathrm{f} 10=0 \times 123456, \\
\% \mathrm{f} 12=0 \times \text { aaaaaa })\end{array}$ & 176 & \\
\hline 44 & $38 \& 43$ & & 212 & 28 \\
\hline
\end{tabular}

the instructions in the sequences is shown in Column 2 and the $I 1$ current cost is shown in Column 3 ( $I 2$ current was $21 \mathrm{~mA}$ in all cases). The maximum variation in the current is only $4 \mathrm{~mA}$, or about $1.9 \%$. Since all the sequences take the same number of cycles, the energy variation is also $1.9 \%$.

Similar observations were also made in the case of the Intel 486DX2 [13]. It appears that this is characteristic of large, complex CPUs, where a major part of the circuit activity is common to all instructions, e.g., instruction fetch, pipeline control, clocks, etc. However, it may be the case that instruction reordering can result in significant variation in smaller processors (as seen in the case of a DSP [7]), and processors with complex power management features. This bears further investigation. 
TABLE VII Example of instruction reordering

\begin{tabular}{|c|c|c|}
\hline No. & Instruction & Register contents \\
\hline $\begin{array}{l}1 \\
2 \\
3 \\
4 \\
5 \\
6 \\
7\end{array}$ & $\begin{array}{l}\text { fmuls } \% \text { f } 8, \% \text { f } 4, \% \text { f } 0 \\
\text { andcc } \% \text { g1, 0xaaa, \%10 } \\
\text { faddd \%10, \%f } 12, \% \text { f } 14 \\
\text { ld [0x555], \%o5 } \\
\text { sll \%o4, ox } 7, \% \text { o6 } \\
\text { sub \%i3, \%i4, \%15 } \\
\text { or } \% g 0,0 x f f, \% 10\end{array}$ & $\begin{array}{l}(\% \mathrm{f} 8=0, \% \mathrm{f} 4=0) \\
(\% \mathrm{~g} 1=0 \times 555) \\
(\% \mathrm{f} 10=0 \times 123456, \% \mathrm{f} 12=0 \times \mathrm{xaaaaaa}) \\
(\% \mathrm{o} 4=0 \times 707) \\
(\% \mathrm{i} 3=0 \times 7 \mathrm{f}, \% \mathrm{i} 4=0 \times 44)\end{array}$ \\
\hline & Sequence & $I 1(\mathrm{~mA})$ \\
\hline $\begin{array}{l}\mathrm{a} \\
\mathrm{b} \\
\mathrm{c} \\
\mathrm{d} \\
\mathrm{e}\end{array}$ & $\begin{array}{l}1,2,3,4,5,6,7 \\
1,3,5,7,2,4,6 \\
1,4,7,2,5,3,6 \\
2,3,7,6,1,5,4 \\
5,3,1,4,6,7,2\end{array}$ & $\begin{array}{l}206.5 \\
203 \\
205 \\
207 \\
202.5\end{array}$ \\
\hline
\end{tabular}

\subsection{Pipeline Stalls and Cache Misses}

The '934 is a pipelined processor. Resource constraints in the pipeline can lead to pipeline stalls that affect the energy cost of programs. Examples are prefetch buffer stalls and write buffer stalls. Base costs of instructions do not reflect the impact of these stalls. Therefore, current measurement experiments are conducted to isolate the power cost of these stalls. The basic idea is to write programs where these stalls occur repeatedly. This is illustrated in greater detail in the context of the Intel 486DX2 in [14]. The energy cost of each kind of stall is proportional to the product of the average current during the stall and the number of cycles involved in the stall, as given by the formula in Section 3.

Another inter-instruction effect that is not reflected in the base costs is the impact of cache misses. Base costs are determined in the context of cache hits in both the instruction and data caches. Cache misses are modelled separately as an energy overhead. This is analogous to what is done for estimating execution time, where a performance penalty is incurred for each cache miss. The results in Section 6 give an idea of the power cost of a cache miss. These costs can be further isolated by conducting experiments with programs where cache misses occur repeatedly. The energy penalty for a cache miss is proportional to the product of the average current during a cache miss and the number of cycles for which the CPU is stalled.

\section{INSTRUCTION LEVEL POWER MODEL}

The results of the previous section quantify the parameters of the instruction-level energy model of the '934. It consists of base energy costs of individual instructions, and energy costs of effects that involve more than one instruction, e.g., effects of circuit state, pipeline, and write buffer stalls. This model forms the basis for estimating the energy cost of given programs. The following simple example illustrates the validity of this model.

\subsection{Program Energy Estimation Example}

Entries 1 to 4 in Table VIII show a program consisting of a sequence of four instructions. The caches were enabled but the entries were locked in. Columns 4 and 5 show the base current costs of instructions. Instruction 4 takes 2 cycles to execute while instructions 1 to 3 take 1 each. The measured current for the full sequence is also shown. The first step is to estimate the cost for the sequence using just the base costs. Multiplying the $I 1$ currents by the number of cycles for each instruction, summing these up, and then dividing 
TABLE VIII Program energy estimation example

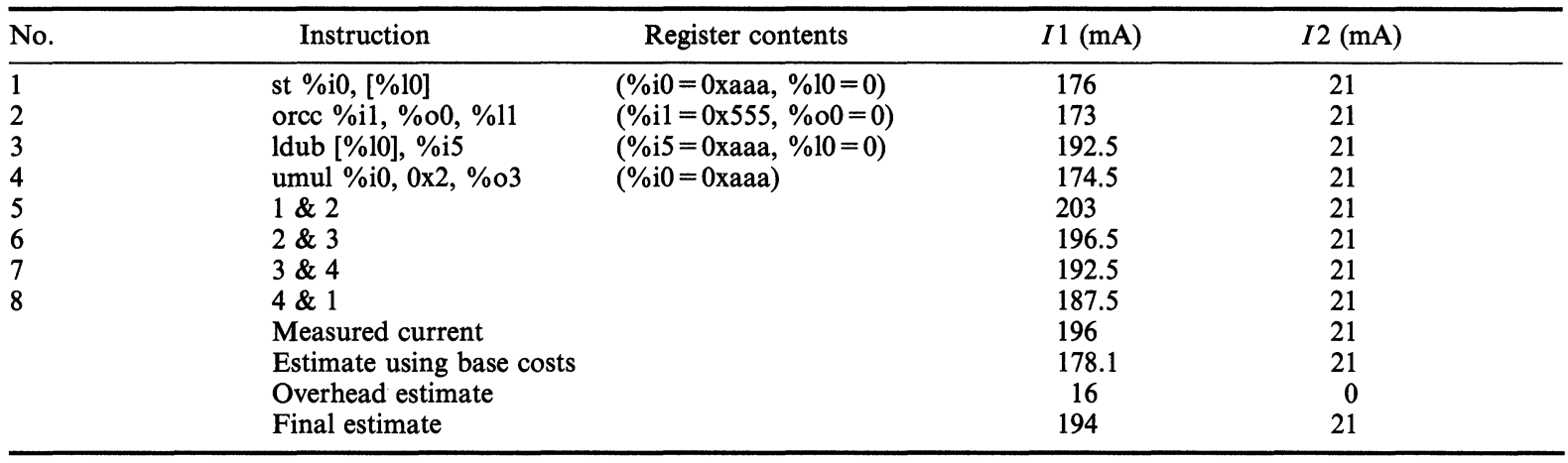

by the total number of cycles, gives an estimate for the $I 1$ current for the sequence:

$$
\begin{aligned}
& (176.0 * 1+173.0 * 1+192.5 * 1+174.5 * 2) / 5 \\
& \quad=178.1 \mathrm{~mA}
\end{aligned}
$$

The estimate for $I 2$ is obviously $21 \mathrm{~mA}$. The interinstruction effects should now be accounted for, which in this example only include the effect of circuit state. This effect is modeled by considering the circuit state overhead between each pair of consecutive instructions. The measured cost for each pair is shown in entries 6 to 8 . The circuit state overhead between instructions 2 and 3 is given by $(196.5-(173.0+192.5) / 2)=13.75 \mathrm{~mA}$. Between 3 and 4 it is given by (192.5$(192.5+2 * 174.5) / 3) * 3 / 2=18.0 \mathrm{~mA}$. The reason for multiplying by $3 / 2$ is that for an alternating sequence of instructions $3 \& 4$, the overhead occurs twice during 3 cycles. In a similar way, the overheads between $1 \& 2$ and $4 \& 1$ are seen to be $28.5 \mathrm{~mA}$ and $18.75 \mathrm{~mA}$, respectively. The average overhead is $16 \mathrm{~mA}$ for $I 1$ and $0 \mathrm{~mA}$ for $I 2$. When these overheads are added to the base cost estimates, we obtain $194 \mathrm{~mA}$ for $I 1$ and $21 \mathrm{~mA}$ for $I 2$. A comparison of entries 9 and 12 shows the close correspondence of the estimate predicted by the instruction-level power model and the actual estimate. Such a close correspondence was also obtained for other experiments involving other instruction sequences.
The overall instruction level power model for the ' 934 is summarized below. Given a program, $P$, its overall energy cost, $E_{P}$ is given by:

$$
E_{P}=\sum_{i}\left(B_{i} \times N_{i}\right)+\sum_{i, j}\left(O_{i, j} \times N_{i, j}\right)+\sum_{k} E_{k}
$$

where for each instruction $i, B_{i}$ is the base cost, and $N_{i}$ is the number of times it will be executed, and for each pair of consecutive instructions $(i, j), O_{i, j}$ is the circuit state overhead, and $N_{i, j}$ is the number of times the pair will be executed. $E_{k}$ is the energy contribution of the other inter instruction effects, $k$ (stalls and cache misses), that would occur during the execution of the program.

The base cost values $\left(B_{i}\right)$ are obtained as shown in the previous sections. The circuit state overhead values $\left(O_{i, j}\right)$ for all possible instruction pairs are also obtained as shown in Section 10. However, given that the circuit state overhead varies in a limited range in the case of the '934, it suggests that a constant value could be used for all instruction pairs. This is a more efficient and yet fairly accurate way of modelling this effect. A value of $18 \mathrm{~mA}$ has been found to be suitable.

The other parameters in the above formula vary from program to program. The execution counts $N_{i}$ and $N_{i, j}$ depend on the execution path of the program. This is dynamic, run-time information. In certain cases it can be determined statically but in general it is best obtained from a program 
profiler. For estimating $E_{k}$, the number of times pipeline stalls and cache misses occur has to be determined. This is again dynamic information that can be statically predicted only in certain cases. In general, this information is obtained from a program profiler and cache simulator.

The basic energy model developed in the previous sections and described above is remarkably similar to the model for the Intel 486DX2. Therefore, the software power/energy estimation framework that was developed for the 486DX2 [14] can be directly applied to the ' 934 .

\section{SOFTWARE CONTROLLED PARTIAL POWER DOWN}

One of the unique features of the ' 934 is a facility for powering down certain CPU modules that are not needed. This is achieved by setting the appropriate bits in a system control register known as the Power-Down Register. The modules that can be powered down are the SDRAM interface (SDI), the DMA module, the floating-point unit (FPU) and the floating-point FIFOs. Bits in the Power-Down Register that are set to 1 cause the clock input of the corresponding module to be disabled. Clearing the bits re-enables the clock input.

Table IX shows the results of experiments that study the effect of powering down specific modules, or combinations of modules. The $I 1$ currents for two different instructions, an OR and a LD are shown in Columns 3 and 5, respectively. The percent reduction in current for each entry is shown in Columns 4 and 6. Entry 1 shows the results when nothing is powered down. Powering down different modules leads to different power savings, the maximum being the case when all the four modules, SDI, DMA, FIFO, and FPU are powered down.

\section{Observations and Comments}

- As can clearly be seen, powering down of unneeded modules can lead to significant power savings. However, powering down and later powering up a module through software, involves the execution of a certain number of control instructions. These instruction themselves consume energy. Thus, powering down of modules will lead to energy savings only if the modules are powered down long enough to compensate for the overhead involved in powering them down and then up.

- The previous observation also indicates that automatic power management of modules will be more effective in saving power. The overhead of powering up and down will be negligible if it is controlled by logic internal to the CPU. Plus, the temporal resolution of the power management strategy can then be much finer - it can even be performed on a cycle by cycle basis.

TABLE IX Software controlled partial power down

\begin{tabular}{|c|c|c|c|c|c|}
\hline \multirow[t]{2}{*}{ 3-6 No. } & \multirow[t]{2}{*}{ Powered-down units } & \multicolumn{2}{|c|}{ or $\% \mathrm{i} 0,0, \% 10$} & \multicolumn{2}{|c|}{ ld [\%10], \%i0 } \\
\hline & & $I 1(\mathrm{~mA})$ & $\%$ saved & $I 2(\mathrm{~mA})$ & $\%$ saved \\
\hline 1 & None & 177 & 0.0 & 192.5 & 0.0 \\
\hline 2 & SDI & 164 & 7.6 & 188.5 & 2.1 \\
\hline 3 & DMA & 164 & 7.6 & 188 & 2.3 \\
\hline 4 & FPU & 155.5 & 12.1 & 180 & 6.5 \\
\hline 5 & FIFO & 155.5 & 12.1 & 180 & 6.5 \\
\hline 6 & DMA, FPU & 151.5 & 14.4 & 174 & 9.6 \\
\hline 7 & DMA, FIFO & 151.5 & 14.4 & 175 & 9.1 \\
\hline 8 & FIFO, FPU & 142 & 19.8 & 166 & 13.8 \\
\hline 9 & DMA, FIFO, FPU & 138 & 22.0 & 162.5 & 15.6 \\
\hline 10 & SDI, DMA, FIFO, FPU & 133 & 25.0 & 157 & 18.4 \\
\hline
\end{tabular}


- An interesting observation leading from the above table is that the power saving achieved by powering down a combination of modules doesn't necessarily equal the sum of the individual power reductions. For example, for the OR instruction, power savings in entry 10 are not equal to the sum of the savings shown in entries 2 to 5 . This is because circuit activity in different modules might be correlated for this instruction. Powering down one module also eliminates some activity in another module. Thus, powering down these modules together results in different savings than what is expected from the savings achieved by powering then down individually.

This actually illustrates the fact that power estimation/analysis methods have to account for correlations between the activities in various modules for each instruction. A power estimation method based on summing up typical power consumptions of separate modules, while disregarding correlations, can be very inaccurate. Thus, the exact correlations have to be known in order to effectively use such a method. The alternative is to use methods which estimate/analyze the power consumption of the entire CPU as a whole. The measurement based analysis method described in this paper is such a method. It implicitly accounts for all correlations between internal modules, since it is based on measurements made at the boundaries of the processor.

\section{CONCLUSIONS}

This paper describes the application of a new power analysis technique for analyzing the power consumption of the Fujitsu SPARClite MB86934, a RISC processor. This technique had earlier been applied to the Intel $486 \mathrm{DX} 2$, a CISC processor. The successful application of this technique for both these processors points to its general applicability for other processors. This study reveals that the basic instruction-level power model of the '934 is very similar to that of the $486 \mathrm{DX} 2$. This power model can be used to effectively evaluate the power cost of software, without requiring knowledge of the proprietary lower level details of the processor. The results of the analysis also provide valuable information about the power consumption in the '934. Besides suggesting several ideas for the design of power efficient software, this information reveals other avenues for power reduction in the processor's design.

\section{Acknowledgment}

The authors would like to thank that D. Maheshwari, H. Kotcherlakota, M. Somasundaram, A. Watanabe, and B. McKeever of Fujitsu Microelectronics Inc. for their help with the experimental setup and for technical discussions.

\section{References}

[1] Feigel, C. and Enfield, M., Fujitsu extends SPARClite family. Microprocessor Report, June 1994.

[2] Fujitsu Microelectronics Inc. SPARClite Embedded Processor User's Manual, 1993.

[3] Fujitsu Microelectronics Inc. SPARClite Embedded Processor User's Manual: MB86934 Addendum, 1994.

[4] Huang, C. X., Zhang, B., Deng, A. C. and Swirski, B., The design and implementation of PowerMill. In Proceedings of the International Symposium on Low Power Design, pp. 105-110, Dana Point, CA, April 1995.

[5] Landman, P. and Rabaey, J., Black-box capacitance models for architectural power analysis. In Proceedings of the International Workshop on Low Power Design, pp. 165-170, Napa, CA, April 1994.

[6] Landman, P. and Rabaey, J., Activity-sensitive architectural power analysis for the control path. In Proceedings of the International Symposium on Low Power Design, pp. 93-98, Dana Point, CA, April 1995.

[7] Lee, T. C., Tiwari, V., Malik, S. and Fujita, M., Power analysis and low-power scheduling techniques for embedded DSP software. In Proceedings of the International Symposium on System Synthesis, Cannes, France, Sept. 1995.

[8] Nagle, L. W. (1975). SPICE2: A computer program to simulate semiconductor circuits. Technical Report ERLM520, University of California, Berkeley.

[9] Salz, A. and Horowitz, M. (1989). IRSIM: An incremental MOS switch-level simulator. In Proceedings of the Design Automation Conference, pages 173-178.

[10] Sato, T., Nagamatsu, M. and Tago, H., Power and performance simulator: ESP and its application for 100MIPS/W class RISC design. In Proceedings of 1994 IEEE Symposium on Low Power Electronics, pp. 46-47, San Diego, CA, Oct. 1994. 
[11] Lu, C. L., Tsui, C. Y. and Despain, A. M., Low power architecture design and compilation techniques for highperformance processors. In IEEE COMPCON, Feb. 1994.

[12] Tiwari, V., Malik, S. and Ashar, P., Guarded evaluation: Pushing power management to logic synthesis/design. In Proceedings of the International Symposium on Low Power Design, pp. 221-226, Dana Point, CA, April 1995.

[13] Tiwari, V., Malik, S. and Wolfe, A., Compilation techniques for low energy: An overview. In Proceedings of 1994 IEEE Symposium on Low Power Electronics, pp. 38-39, San Diego, CA, Oct. 1994.

[14] Tiwari, V., Malik, S. and Wolfe, A., Power analysis of embedded software: A first step towards software power minimization. IEEE Transactions on VLSI Systems, 2(4), 437-445, December 1994.

[15] Ong, P. W. and Yan, R. H., Power-conscious software design- a framework for modeling software on hardware. In Proceedings of 1994 Symposium on Low Power Electronics, pp. 36-37, San Diego, CA, Oct. 1994.

\section{Authors' Biographies}

Vivek Tiwari received the B.Tech., degree in Computer Science and Engineering from the Indian Institute of Technology, New Delhi, India in 1991. Currently he is working towards the $\mathrm{Ph} . \mathrm{D}$. degree in the Department of Electrical Engineering, Princeton University.

His research interests are in the areas of Computer Aided Design of VLSI and embedded systems and in microprocessor architecture. The focus of his current research is on tools and techniques for power estimation and low power design. He has held summer positions at NEC Research Labs (1993), Intel Corporation (1994), Fujitsu Labs of America (1994), and IBM T. J. Watson Research Center (1995), where he worked on the above topics.

He received the IBM Graduate Fellowship Award in 1993, 1994, and 1995, and a Best Paper Award at ASP-DAC' 95.

Mike Tien-Chien Lee received his B.S., degree in Computer Science from National Taiwan University in 1987, and the M.S., degree and the Ph.D., degree in electrical engineering from Princeton University, in 1991 and 1993, respectively.

He is currently a researcher at Fujitsu Laboratories of America, Santa Clara, CA. His research interests include low-power design, embedded system design, high-level synthesis, and test synthesis.

$\mathrm{He}$ has served in the program committees of IEEE Pacific Northwest Test Workshop and IEEE International Test Synthesis Workshop. He is also a consulting researcher at Center of Reliable Computing, Stanford University. He received a Best Paper Award at ASP-DAC'95. 

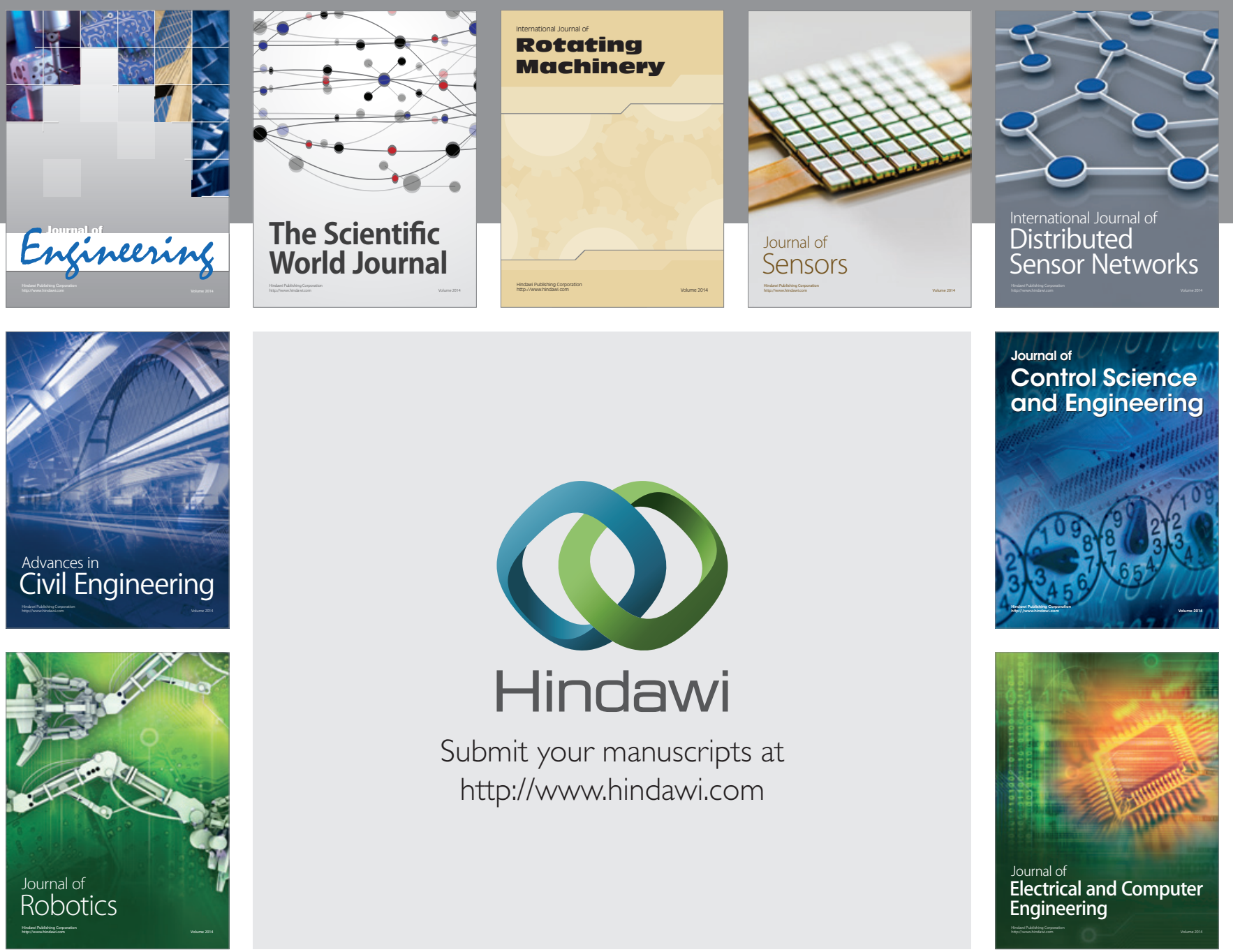

Submit your manuscripts at

http://www.hindawi.com
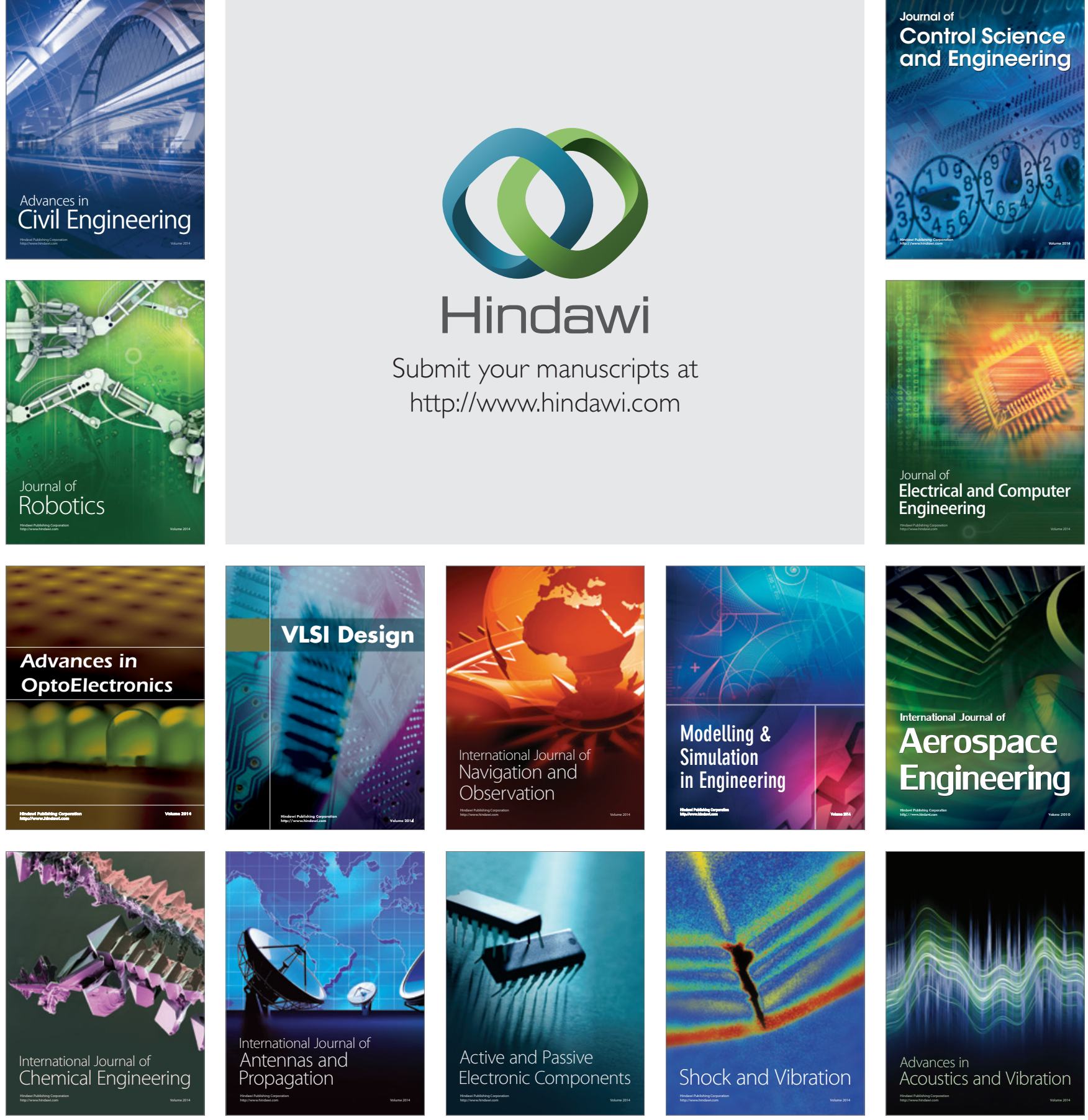